\title{
Resistant Temporal Lobe Epilepsy: Initial Steps into a Bigger Epilepsy Surgery Program
}

\author{
Anant Mehrotra ${ }^{1}$ Suyash Singh ${ }^{1}$ Soumen Kanjilal ${ }^{1}$ Gagandeep Attri ${ }^{1}$ Kamlesh Rangari ${ }^{1}$ \\ Vimal K. Paliwal $\quad$ Vinita Mani² Pawan K. Verma ${ }^{1}$ Ved Prakash Maurya ${ }^{1}$ Jayesh Sardhara ${ }^{1}$ \\ Kamlesh S. Bhaisora ${ }^{1} \quad$ Kuntal Kanti Das ${ }^{1}$ Arun Kumar Srivastava ${ }^{1} \quad$ Awadhesh K. Jaiswal ${ }^{1}$
}

Sanjay Behari ${ }^{1}$

1Department of Neurosurgery, Sanjay Gandhi Post Graduate
Institute of Medical Sciences, Lucknow, Uttar Pradesh, India
2Department of Neurology, Sanjay Gandhi Post Graduate Institute
of Medical Sciences, Lucknow, Uttar Pradesh, India

J Neurosci Rural Pract:2021;12:193-196

\begin{abstract}
Address for correspondence Anant Mehrotra, MS, MCh, Department of Neurosurgery, Sanjay Gandhi Post Graduate Institute of medical Sciences, Lucknow, Uttar Pradesh, India (e-mail: dranantmehrotra@gmail.com).
\end{abstract}

\begin{abstract}
Keywords

- drug-resistant epilepsy

- temporal lobe epilepsy

- anterior-medial temporal lobe resection

- international league against epilepsy
\end{abstract}

Background Among the patients of drug-resistant epilepsy, a subset which has focal impaired seizures localizes to the temporal lobe region (TLE). A majority of these cases are surgically amenable with anterior-medial temporal lobe resection or "lesionectomy."

Objective In India, there is scarcity of "specialized centers" providing "comprehensive epilepsy care" and this dearth is further worse in populous states. In this article, we share our single center, observational, and retrospective experience of TLE in background of limited resources and utmost requirement.

Methodology Our study is a retrospective analysis medically refractory epilepsy patients (2016-2019). Patients with medically refractory epilepsy were selected based upon our noninvasive protocol (clinical semiology, interictal scalp electroencephalography (EEG), long-term video EEG monitoring data, and magnetic resonance injury [MRI]). The follow-up was noted from the last out-patient visit record or through telephonic conversation (International League Against Epilepsy score).

Results Of 23 cases of TLE ( $n=7$, mesial temporal sclerosis; $n=16$ temporal lobe like cavernomas, tumors, or arterio-venous malformations). Single photon emission computed tomography/positron emission tomography (SPECT/PET) was performed in five cases (three cases of ictal/interictal SPECT and two cases of PET scan) where there was discordance between EEG/clinical and MRI. The median follow-up was of 19 months with 18 cases being seizure free. Five cases were fully off the antiepileptic drug (AEDs) while in 15 cases, the AEDs dosages or the number were reduced. Average number of AEDs reduced from 2.9 in preoperative period to 1.2 postoperatively. Two cases had quadrantanopia and one case of cerebrospinal fluid leak.

Conclusion A multidisciplinary and holistic approach is required for best patient care. The results of our initial surgical experience are encouraging.
DOI https://doi.org/ $10.1055 / \mathrm{s}-0040-1716796$ ISSN 0976-3147.

\footnotetext{
(c) 2020. Association for Helping Neurosurgical Sick People.

This is an open access article published by Thieme under the terms of the Creative Commons Attribution-NonDerivative-NonCommercial-License, permitting copying and reproduction so long as the original work is given appropriate credit. Contents may not be used for commercial purposes, or adapted, remixed, transformed or built upon. (https://creativecommons.org/licenses/by-nc-nd/4.0/) Thieme Medical and Scientific Publishers Pvt. Ltd., A-12, 2nd Floor, Sector 2, Noida-201301 UP, India
} 


\section{Introduction}

Twenty-five percent of the epilepsy patients may subsequently develop drug-resistant epilepsy (DRE)., ${ }^{1,2}$ Among these patients, the majority have focal unaware seizures, which localizes to the temporal lobe region (TLE). ${ }^{2}$ These subset of patients are grouped under TLE. Among the spectrum of lesions in the temporal lobe, (like tumors, cavernomas, and arterio-venous malformations), mesial temporal sclerosis (MTS) is the most common cause of TLE. ${ }^{1-3}$ Fortunately, a majority of MTS cases are surgically amenable and further depending on their location either "anterior-medial temporal lobe resection" (AMTR) or "lesionectomy only" is offered. The surgical outcome of TLE is good and provided there is concordance between clinical semiology and radiological findings. An advanced and costly investigation like single photon emission computerized tomography (SPECT) or positron emission tomography (PET) are usually not required for a classical case of TLE or specifically MTS. ${ }^{2-4}$ In India, there is scarcity of "specialized centers" providing "comprehensive epilepsy care," and this dearth is further worse in populous states like Uttar Pradesh and Madhya Pradesh (not more than two to three such centers in 2016). To the best of our knowledge, the situation has not improved further. In this article, we share our single center, observational, and retrospective experience of TLE in background of limited resources and utmost requirement.

\section{Methodology}

\section{Study Design}

Our study is a retrospective analysis of the prospectively maintained records of medically refractory epilepsy patients. We segregated all the patients of temporal lobe pathology who underwent surgical treatment at our center (between January 2016 and December 2019). Medically refractory epilepsy or DRE was defined as per the International League Against Epilepsy (ILAE) definition "failure of adequate trials of two tolerated and appropriately chosen and used antiepileptic drug schedules (whether as monotherapies or in combination) achieve sustained seizure freedom." 5

\section{Inclusion and Exclusion Criteria}

Patients with temporal lobe pathology who presented with DRE were included in our study. Patients who presented with symptoms other than seizure or whose medical records could not be retrieved were excluded from this study.

\section{Management Protocol}

Patients were selected based upon our noninvasive protocol, comprising of clinical semiology, interictal scalp EEG, long-term video EEG monitoring data, and magnetic resonance imaging (MRI). SPECT and PET were performed in the cases where we found discordance between clinical/ EEG data and MRI findings. The decision regarding surgical treatment was based upon the type of lesion, location of the lesion (especially its relation to the eloquent cortex), and finally concluded by a panel of experts (neurosurgeons, neurologists, and neuro-anesthesiologist). For MTS or other "medially placed tumors in the temporal lobe," an AMTR was performed (along with resection of tumor when indicated). For the "laterally placed temporal lobe tumors," the standard microsurgical resection techniques were used along with intraoperative EEG for the resection epileptic focus. The anterior two-third of the hippocampus was resected en bloc and lateral half to two-third of the amygdala was resected along with parahippocampal gyrus and uncus. The follow-up was noted from the last out-patient visit record or through telephonic conversation. The ILAE score was used to assess the outcome, and the perioperative complications were also noted. $^{2}$

\section{Results}

Sixty-one patients were initially put up under panel of DRE, wherein 30 cases were excluded. Among these 30 cases, 14 cases were receiving inadequate dosages of antiepileptic drugs, five cases were diagnosed as case of pseudo-seizure, eight cases were diagnosed as cases of primary generalized tonic clonic seizures, two patients refused any surgical intervention, and in one case no proper localization was possible. All these cases were managed with appropriate antiepileptic drug (AEDs) dosages. We have also excluded the patients of temporal lobe lesions, who did not undergo a detailed presurgical epilepsy evaluation or those cases who presented with features of mass effect and not DRE as the primary presentation.

Among the remaining 31 cases, 23 patients of temporal lobe epilepsy (TLE) (Male:Female $=18: 5$ ) and eight patients of extratemporal lobe epilepsy were further studies. Among the 23 cases of TLE, 15 cases had focal unaware seizures, three cases had focal aware seizures, and five cases had focal aware seizures with bilaterally tonic-clonic seizures. One patient of hippocampal AVM had history of drug-resistant epilepsy but was referred to us after an event of AVM bleed. The median duration of symptoms was 68 months. Six patients were below 10 years of age, with maximum cases in the age group between 21 to 30 years $(n=10)$. Three patients were between 31 to 40 years and remaining four cases were above 40 years of age.

Among the 23 cases of TLE, seven were cases of mesial temporal sclerosis (MTS), while the remaining 16 cases were of temporal lobe like cavernomas, tumors, or arterio-venous malformations. If needed, intraoperative neuro-monitoring including intraoperative EEG was used. The list of pathological diagnosis is seen in -Fig. $\mathbf{1}$ with low-grade gliomas being the most common lesion followed by MTS. SPECT/PET was performed in five cases (three cases of ictal/interictal SPECT and two cases of PET scan), where there was discordance between EEG/clinical findings and MRI or MRI was inconclusive.

The median follow-up of these cases was of 19 months. Out of the 23 cases, 18 cases were seizure free and in the remaining five cases, the seizure frequency was reduced. Five cases were fully off the AEDs, while in 15 cases the AEDs dosages or the number of drugs being taken or both were 


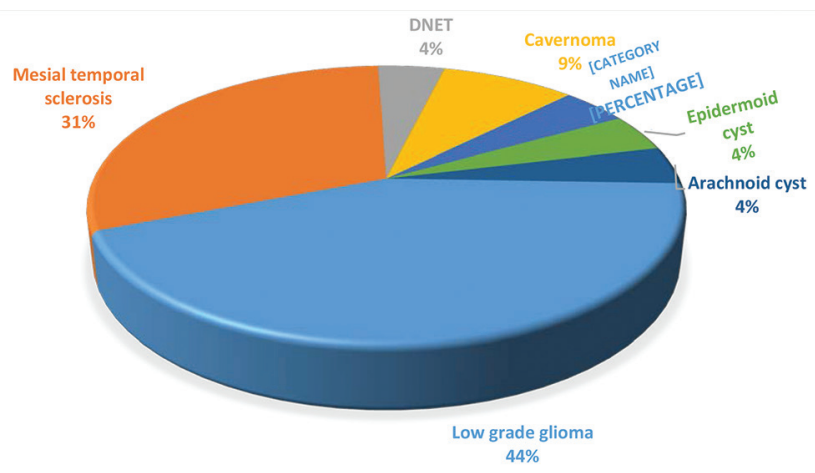

Fig. 1 Three-dimensional pie chart showing various pathologies in our series.

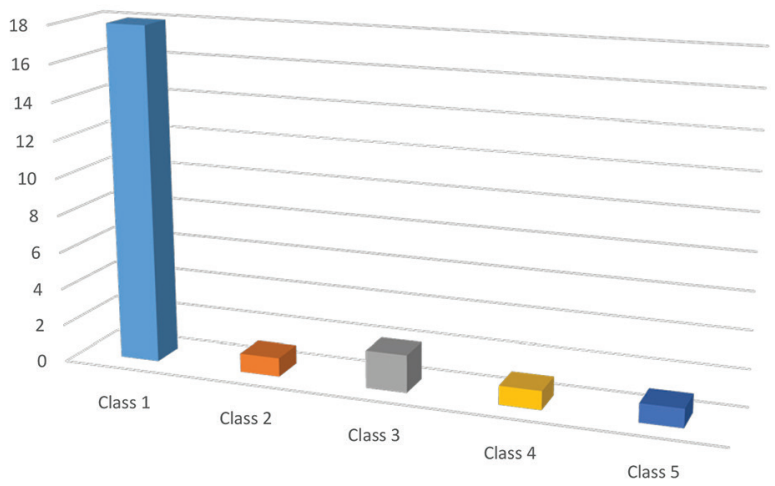

Fig. 2 Graphical representation showing surgical outcome according to International League Against Epilepsy.

reduced. In the remaining three patients, the AEDs were continued in preoperative dosages. Average preoperative AEDs advised to a patient was 2.9 which was reduced to 1.2 in the postoperative period. The outcome as per the ILAE score is shown in -Fig. 2 with 18 patients in class I and no patient in class VI. There was one case of cerebro-spinal fluid (CSF) leak from the wound which was conservatively managed. Two cases had superior quadrantanopia, but it was an examination finding and the patients were not aware of it. Although a detailed neuro-psychological testing was not done in the postoperative period, none of our patients complained of any significant memory deficits.

\section{Discussion}

Comprehensive care of epilepsy is available in only a selected few centers of India. There is a big gap between the demand of epilepsy surgeries needed and the number of epilepsy surgery being performed in our country. The situation is even worse in more populous states of our country like Uttar Pradesh and Madhya Pradesh. As in 2016, these two states were home to only two and one epilepsy centers, respectively.

The interdisciplinary and collaborative efforts of neurology, neurosurgery, neuro-radiology, and neuro-anesthesia departments forms the essential pillar of an epilepsy center. Our state has a population of 23 crores (https://www. census2011.co.in/census/state/uttar+pradesh.html) and our center caters patients from three to four nearby states also. The growing need of a dedicated epilepsy surgery unit encouraged us to sustain all the initial hardships in developing such facility for patients. This paper highlights our initial journey and experience through all oppressions and adversities. Our center is a relatively new epilepsy center and we have performed 31 cases of epilepsy surgery since 2016.

TLE is the most common indication for epilepsy surgery. In the study by Wiebe et al, 80 patients were randomized to surgical and medical group with 40 cases in each group. ${ }^{6}$ At 1 -year of follow-up, $58 \%$ of cases in surgical group as compared with $8 \%$ of cases in medical group were seizure free. Patients in the surgical group had fewer seizures impairing awareness and also had a better quality of life. ${ }^{6}$ Similar results have been reported in other series as well..$^{7-10}$

In our series, we operated upon 23 cases of TLE. Out of these 23 cases, 18 cases were seizure free. In our series $78 \%$ of cases were seizure free, and this percentage is higher than the results seen in some other series which report a seizure-free cases in 65 to $77 \%$ of cases. ${ }^{8,11,12}$ This number might be reduced further with a longer follow-up. Our results were similar to results seen by Mohamed et al, where they analyzed the surgical outcome in 34 cases of TLE due to hippocampal sclerosis. ${ }^{13}$ In total, $78 \%$ of their cases were seizure free at a mean follow-up of 2.6 years. Our results might change in future as the follow-up in our study is only 19 months.

The average number of AEDs that was prescribed in the preoperative period was 2.9 and in the postoperative period, this was reduced to 1.2 AEDs per patient. Five of our patients (21.7\%) were completely weaned off all AEDs. Rao et al had reported an incidence of $30 \%$ cases which were free of all AEDs in a follow-up of 3 years. ${ }^{3}$ With a longer follow-up, the number of patients who were free of all AEDs might increase.

We had our share of complications, but fortunately none of them were major complications. In all, we had three complications (13\%). We had one CSF leak from wound site which was managed conservatively, and two patients had superior quadrantanopia, but both these cases were unaware of the deficit and it was found on routine postoperative check up. The complication rate was slightly lower than the reported complications by Panigrahi et al who had reported complications in $18.9 \%$ of cases. ${ }^{14}$ The slightly lower complications could be because a detailed postoperative neuro-psychological testing was not performed in all cases, and so subtle deficits might have been missed.

Epilepsy surgery in a new set-up needs to progress in a step-wise pattern for better results. ${ }^{15}$ In future, we plan to expand our horizons of epilepsy surgery, especially establish protocols regarding invasive EEG monitoring so that disconcordant cases can also be offered surgical treatment whenever possible.

\section{Conclusion}

In conclusion, we present our initial experience of epilepsy surgery for TLE along with the results and their complications. 
The results of our initial cases are encouraging and are similar to the reported literature. A multidisciplinary and holistic approach is required for best patient outcome. We believe that as our epilepsy team gains more experience, we will be able to tackle more complicated cases and also will expand our horizons in both TLE and extratemporal lobe epilepsy.

\section{Funding}

None.

\section{Conflict of Interest}

None declared.

\section{References}

1 Bruce M, Griffiths C, Brock A, Majeed A. Trends in mortality and hospital admissions associated with epilepsy in England and Wales during the 1990s. Health Stat Q 2004;21(21):23-29

2 Escudeiro GP, Leal RTM, Almeida HH, Acioly MA, Landeiro JA, Pessoa BL. Implementing an epilepsy surgery center. Initial experience at a university hospital in Brazil. Arq Neuropsiquiatr 2017;75(10):722-726

3 Rao MB, Radhakrishnan K. Is epilepsy surgery possible in countries with limited resources.? Epilepsia 2000;41(Suppl 4): S31-S34

4 Rathore C, Radhakrishnan K. Epidemiology of epilepsy surgery in India. Neurol India 2017;65(Supplement) :S52-S59

5 Kwan P, Arzimanoglou A, Berg AT, et al. Definition of drug resistant epilepsy: consensus proposal by the ad hoc Task Force of the ILAE Commission on Therapeutic Strategies. Epilepsia 2010;51(6):1069-1077

6 Wiebe S, Jetté N. Epilepsy surgery utilization: who, when, where, and why? Curr Opin Neurol 2012;25(2):187-193

7 Bonilha L, Martz GU, Glazier SS, Edwards JC. Subtypes of medial temporal lobe epilepsy: influence on temporal lobectomy outcomes? Epilepsia 2012;53(1):1-6

8 Téllez-Zenteno JF, Dhar R, Wiebe S. Long-term seizure outcomes following epilepsy surgery: a systematic review and meta-analysis. Brain 2005;128(Pt 5) :1188-1198

9 de Tisi J, Bell GS, Peacock JL, et al. The long-term outcome of adult epilepsy surgery, patterns of seizure remission, and relapse: a cohort study. Lancet 2011;378(9800):1388-1395

10 Berg AT, Langfitt J, Shinnar S, et al. How long does it take for partial epilepsy to become intractable? Neurology 2003;60(2):186-190

11 Dwivedi R, Ramanujam B, Chandra PS, et al. Surgery for drug-resistant epilepsy in children. N Engl J Med 2017;377(17): 1639-1647

12 Radhakrishnan A, Menon R, Menon D, et al. Early resective surgery causes favorable seizure outcome in malformations of cortical development. Epilepsy Res 2016;124:1-11

13 Mohamed A, Wyllie E, Ruggieri P, et al. Temporal lobe epilepsy due to hippocampal sclerosis in pediatric candidates for epilepsy surgery. Neurology 2001;56(12):1643-1649

14 Panigrahi M, Vooturi S, Jayalakshmi S. Complications of epilepsy surgery: a single surgeon's experience from South India. World Neurosurg 2016;91:16-22

15 Chandra PS, Tripathi M. Epilepsy surgery: recommendations for India. Ann Indian Acad Neurol 2010;13(2):87-93 